\title{
ORIGINAL
}

\section{Effects of chlorhexidine gluconate oral care on hospital mortality: a hospital-wide, observational cohort study}

\author{
Mieke Deschepper ${ }^{1}$, Willem Waegeman², Kristof Eeckloo ${ }^{1,3}$, Dirk Vogelaers ${ }^{4,5}$ and Stijn Blot $^{5,6^{*}}$ (1)
}

(C) 2018 The Author(s)

\begin{abstract}
Purpose: Chlorhexidine oral care is widely used in critically and non-critically ill hospitalized patients to maintain oral health. We investigated the effect of chlorhexidine oral care on mortality in a general hospitalized population.

Methods: In this single-center, retrospective, hospital-wide, observational cohort study we included adult hospitalized patients (2012-2014). Mortality associated with chlorhexidine oral care was assessed by logistic regression analysis. A threshold cumulative dose of $300 \mathrm{mg}$ served as a dichotomic proxy for chlorhexidine exposure. We adjusted for demographics, diagnostic category, and risk of mortality expressed in four categories (minor, moderate, major, and extreme).

Results: The study cohort included 82,274 patients of which 11,133 (14\%) received chlorhexidine oral care. Lowlevel exposure to chlorhexidine oral care $(\leq 300 \mathrm{mg}$ ) was associated with increased risk of death [odds ratio (OR) 2.61; 95\% confidence interval (Cl) 2.32-2.92]. This association was stronger among patients with a lower risk of death: OR 5.50 (95\% Cl 4.51-6.71) with minor/moderate risk, OR 2.33 (95\% Cl 1.96-2.78) with a major risk, and a not significant OR 1.13 (95\% Cl 0.90-1.41) with an extreme risk of mortality. Similar observations were made for high-level exposure (>300 mg). No harmful effect was observed in ventilated and non-ventilated ICU patients. Increased risk of death was observed in patients who did not receive mechanical ventilation and were not admitted to ICUs. The adjusted number of patients needed to be exposed to result in one additional fatality case was 47.1 ( $95 \% \mathrm{Cl}$ 45.2-49.1).
\end{abstract}

Conclusions: These data argue against the indiscriminate widespread use of chlorhexidine oral care in hospitalized patients, in the absence of proven benefit in specific populations.

Keywords: Chlorhexidine oral care, In-hospital mortality, Risk of mortality

\section{Introduction}

Ventilator-associated pneumonia remains an important threat to critically ill patients as it is associated with considerable morbidity, and possibly mortality, thereby contributing to a substantial economic burden [1-3]. Preventive strategies mainly target optimization of basic infection control measures, avoidance of delayed

\footnotetext{
*Correspondence: stijn.blot@UGent.be

${ }^{5}$ Department of Internal Medicine, Ghent University, Campus UZ Gent, Corneel Heymanslaan 10, 9000 Ghent, Belgium

Full author information is available at the end of the article
}

extubation, biofilm formation on the endotracheal tube and of micro-aspiration of subglottic secretions, prevention of bacterial translocation from stomach to oropharynx, and modulation of oropharyngeal colonization [4-8]. Regarding the last of these, the value of chlorhexidine oral care has been studied extensively. A meta-analysis including 12 randomized studies encompassing 2341 patients reported a significant overall risk reduction in ventilator-associated pneumonia with chlorhexidine oral care [risk ratio (RR) 0.72 , 95\% confidence interval (CI) $0.55-0.94]$ [9]. The strongest effects were observed in cardiac surgery patients (RR $0.41,95 \%$ CI $0.17-0.98$ ) and 
with higher (2\%) chlorhexidine concentrations (RR 0.53, 95\% CI 0.31-0.91). Consequently, chlorhexidine oral care has become common practice. It is recommended for cardiac surgery patients in guidelines of the Centers of Disease Control and Prevention (CDC) and the American Thoracic Society/Infectious Diseases Society of America (ATS/IDSA) [10,11] and proposed as one of the five components of a core set of interventions in the ventilator bundle defined by the Institute for Healthcare Improvement (IHI) [12]. Although not always supported by evidence, chlorhexidine mouthwashes are nowadays widely used in a variety of patient populations, either critically or non-critically ill [13-15].

Recent studies, however, have incited controversy about chlorhexidine mouthwashes. Klompas et al. performed a meta-analysis involving 16 studies (including 3630 patients) that assessed the value of chlorhexidine oral care in the prevention of nosocomial and ventilator-associated pneumonia [16]. The study indicated that chlorhexidine oral care is associated with a risk reduction for nosocomial pneumonia in cardiac surgery patients (RR $0.56,95 \%$ CI $0.41-0.77$ ) while the risk reduction for ventilator-associated pneumonia in non-cardiac surgery patients was not significant (RR 0.78, 95\% CI 0.60-1.02). Unexpectedly, however, this meta-analysis suggested a trend towards increased mortality among patients receiving chlorhexidine oral care (RR 1.13, 95\% CI 0.99-1.28), an effect exclusively observed in non-cardiac surgery patients. In another meta-analysis, pooling the results from 11 randomized controlled trials in general intensive care units (including 2618 patients), Price et al. found chlorhexidine oral care to be associated with increased mortality (odds ratio 1.25, 95\% CI 1.05-1.50) [17]. Finally, in a retrospective study evaluating associations between ventilator bundle components and outcomes, chlorhexidine oral care appeared to be associated with increased ventilator mortality [hazard ratio (HR) 1.63, 95\% CI 1.15-2.31] [18]. Therefore, the international ERS/ ESICM/ESCMID/ALAT guidelines for the management of ventilator-associated pneumonia has not issued a recommendation until more safety data become available [19].

These observations warrant further investigation of chlorhexidine in oral care. The objective of this study is to assess the effect of chlorhexidine oral care on hospital mortality in a large cohort. As chlorhexidine oral care is frequently used in a variety of hospitalized patients, and no mortality data in non-ICU patients are available, this study is explicitly conducted hospital-wide.

\section{Methods}

\section{Study design and data source}

This is a retrospective, observational cohort study including patients hospitalized at Ghent University Hospital. Variables were extracted from data required by the

\section{Take-home message}

In a general hospital population chlorhexidine oral care is significantly associated with increased mortality. These findings incite reconsideration of the practice of chlorhexidine oral care in patient populations for which an evidence-based benefit is lacking.

Belgian government, including billing data for the obligatory health insurance (Healthcare Fund/National Health Service), and administrative and medical data for the Federal Public Service Health, Food Chain Safety, and Environment (Hospital Discharge Dataset). The study was approved by the ethics committee at Ghent University Hospital (Belgian registration no. B670201731386).

\section{Study variables}

The outcome variable is hospital mortality. The main explanatory variable is chlorhexidine oral care. Chlorhexidine oral care is covered by a protocol prescribing a rinseand-spit approach for autonomous patients and cleaning of the oral cavity by the nurse with chlorhexidine soaked sterile gauze in dependent patients. Chlorhexidine oral care is applied twice daily in general wards and thrice daily in ICUs. We used a dichotomic proxy for chlorhexidine exposure to adjust for the extent of exposure in individual patients, defining low $(\leq 300 \mathrm{mg})$ or high $(>300 \mathrm{mg})$ exposure, respectively. The cutoff of $300 \mathrm{mg}$ corresponds to the content of a standard bottle of $0.12 \%$ chlorhexidine of $250 \mathrm{~mL}$. The average amount of chlorhexidine solution used per oral care provision is about $15 \mathrm{~mL}$ and hence in thrice-daily oral care, use of more than $300 \mathrm{mg}$ translates into an exposure time of more than 5-6 days. This measure of chlorhexidine exposure covers chlorhexidine concentration $(0.05 \%$ or $0.12 \%)$, frequency of oral care provision, and exposure time in a single variable. Indications for chlorhexidine oral care cover a wide spectrum of conditions ranging from pneumonia prevention to stomatitis and halitosis. Furthermore, chlorhexidine as a component of oral care is often applied in dependent patients irrespective of a medical indication.

To correct for confounding the following patient characteristics are considered: age at admission, sex, emergency admission (i.e., admission via emergency room), medical or surgical admission (based on principal diagnosis and procedure), and diagnostic category. Adjustment for diagnostic category is based on 25 classes of Major Diagnostic Category (MDC), grouped according to the primary diagnoses as defined in the International Classification of Diseases, Ninth Revision, Clinical Modification (ICD-9-CM) system [20]. Furthermore, adjustment for risk of mortality is based on the All Patient Refined-Diagnosis Related Groups (APR-DRG) [21]. The APR-DRG methodology has been 
developed to permit outcome analysis across large cohorts and provides a means of relating the type of patients a hospital treats to the costs incurred by the hospital [22]. Patients are allocated to an APR-DRG group on the basis of principal diagnosis and procedures [21]. APR-DRGs extend the basic DRG structure by adding two sets of subclasses to each base APR-DRG, i.e., risk of mortality and severity of illness. These are based on the secondary diagnoses. The risk of mortality metrics are used for risk adjustment within each DRG [23-25]. Risk of mortality is expressed in four categories: minor, moderate, major, and extreme risk.

\section{Patient selection}

The study cohort includes patients hospitalized and discharged between 1 January 2012 and 31 December 2014. Only patients aged 16 years or more at time of admission were taken into account. Hospitalized patients allocated to an APR-DRG category without risk of mortality were not considered. Therefore, we excluded all patients admitted to psychiatric or rehabilitation wards. Additionally, we refined the data set by excluding all MDCs with less than six deceased patients: MDC02 (diseases and disorders of the eye), MDC14 (pregnancy, childbirth, and puerperium), MDC15 (newborns and other neonates with conditions originating in the perinatal period), MDC20 (alcohol/drug use and alcohol/drug induced organic mental disorders). Appendix 1 describes the flowchart of the patient selection process. Multiple episodes of hospitalizations were considered as separate admissions.

\section{Statistical analyses Descriptives}

Descriptive data are expressed as $n$ (\%) or median (25th75th percentile). The Pearson chi-square test was used for the comparison of categorical variables and the Wilcoxon rank-sum test for the comparison of continuous variables.

\section{Logistic regression}

To assess relationships with mortality we used a logistic regression analysis with the logit link function. Specifications of the model are described in Supplementary Appendix 2. The logistic regression models were validated by plotting the receiver operating characteristic (ROC) curve, computing the sensitivity and specificity, and calculating the area under the curve. Therefore we split the cohort into a 2:1 random training and test data set. The model is fit (trained) using the training data set and then assessed by applying the model to the test data set. Results of the logistic regression analyses are reported as odds ratio (OR) and $95 \%$ CI; $p<0.05$ denotes statistical significance.

We used the OR generated by the logistic regression analysis to calculate the "number needed to be exposed" to encounter one additional outcome with adjustment for confounding variables and using the multivariate delta method for calculating 95\% CIs [26].

\section{Effect of chlorhexidine oral care on mortality according to risk of mortality category}

Because the effect of chlorhexidine oral care on the outcome may vary according to the risk of mortality, separate logistic regression models were performed in distinct risk of mortality categories. In these models patients with minor and moderate risk of mortality were merged because of the relatively low outcome events among patients with minor risk of mortality (91 deaths representing $0.17 \%)$.

\section{Effect of chlorhexidine oral care on mortality in patients undergoing cardiothoracic and vascular surgery}

We assessed the impact of chlorhexidine oral care on mortality in a subgroup of cardiothoracic and vascular surgery patients because of two reasons. First, the evidence of a favorable effect of chlorhexidine oral care in the prevention of nosocomial pneumonia is most robust in cardiac surgery patients $[9,16]$. Second, in the metaanalysis by Klompas et al. the trend towards increased mortality could be completely attributed to the use of chlorhexidine oral care in non-cardiac surgery patients, whereas in cardiac surgery patients no effect on mortality was observed (RR $0.88,95 \%$ CI $0.25-3.14$ ) [16]. For this purpose we selected two subgroups of patients. The first group involves patients undergoing major cardiothoracic and vascular surgery, while the second group encompasses the total spectrum of both major and minor cardiothoracic and vascular procedures. For the patient selection on major cardiothoracic and vascular surgery we considered patients with the following APR-DRGs: APR-DRG 160 (major cardiothoracic repair of heart anomaly), APR-DRG 165 (coronary bypass with cardiac catheterization or percutaneous cardiac procedure), APR-DRG 166 (coronary bypass without cardiac catheterization or percutaneous cardiac procedure), and APR-DRG 169 (major thoracic and abdominal vascular procedures). The broader selection involved all surgical patients included in MDC-05 (diseases and disorders of the circulatory system).

\section{Effect of chlorhexidine oral care on mortality in patients with or without mechanical ventilation}

Because chlorhexidine oral care is a particular indication for mechanically ventilated patients we assessed the impact of this practice in patients who did and did not experience mechanical ventilation. Additionally, the group of ventilated patients was dichotomized according to length of mechanical ventilation ( $\leq 96$ or $>96 \mathrm{~h}$ ). We further analyze the non-ventilated patients with 
admission on an ICU department during their hospital stay.

All analyses are performed using R Statistical Software, version 3.3.

\section{Results}

Description of the study cohort and unadjusted

relationships between chlorhexidine oral care

and mortality

During the study period 107,308 patients were hospitalized and discharged. After exclusion of admissions as per protocol the final study cohort included 82,274 patients. Supplementary Appendix Fig. 1 describes the flowchart of the patient selection process.

Overall, 11,133 (14\%) patients received chlorhexidine oral care during their hospitalization, either in a solution of $0.05 \%(n=1175)$ or $0.12 \%(n=9963)$. Table 1 reports patient characteristics stratified for survivors and non-survivors. In this unadjusted analysis the use of chlorhexidine oral care was significantly higher in patients who died. Table 2 describes the mortality rates for patients with and without chlorhexidine oral care stratified for risk of mortality category.

\section{Adjusted relationships between chlorhexidine oral care and mortality \\ Total study cohort}

Table 3 shows the results of the final logistic regression model assessing the effect of chlorhexidine oral care on mortality in the total study cohort, after selecting all relevant variables based on AIC. The use of chlorhexidine oral care is significantly associated with increased risk of death. The ROC curve for the test cohort shows an area under the curve of 0.94 (Supplementary Appendix 3). The adjusted number of patients needed to be exposed to chlorhexidine oral care to result in one additional case fatality is 47.15 (95\% CI $45.19-49.11)$.

Table 1 Patient characteristics of the study cohort stratified for survivors and non-survivors

\begin{tabular}{|c|c|c|c|}
\hline Patient characteristic & Survivors, $N=79,879$ & Non-survivors, $N=2395$ & $p$ value* \\
\hline Sex, male & 41,992 (53\%) & 1414 (59\%) & $<0.001$ \\
\hline Age, years & $56(42-69)$ & $70(60-80)$ & $<0.001$ \\
\hline Age category, years & & & $<0.001$ \\
\hline $17-45$ & $23,849(30 \%)$ & 157 (0.7\%) & \\
\hline $46-75$ & $44,781(56 \%)$ & $1381(58 \%)$ & \\
\hline$>75$ & $11,249(14 \%)$ & $857(36 \%)$ & \\
\hline Length of stay, days & $3(2-8)$ & $10(3-22)$ & $<0.001$ \\
\hline Risk of mortality category & & & $<0.001$ \\
\hline 1. Minor & $53,142(67 \%)$ & $91(4 \%)$ & \\
\hline 2. Moderate & $18,693(23 \%)$ & $461(19 \%)$ & \\
\hline 3. Major & $6609(8 \%)$ & $808(34 \%)$ & \\
\hline 4. Extreme & $1435(1.8 \%)$ & $1035(43 \%)$ & \\
\hline Chlorhexidine oral care & & & $<0.001$ \\
\hline No $(0 \mathrm{mg})$ & 70,187 (88\%) & $954(40 \%)$ & \\
\hline Low ( $\leq 300 \mathrm{mg})$ & $7210(9 \%)$ & $870(36 \%)$ & \\
\hline High (> 300 mg) & $2482(3 \%)$ & $571(24 \%)$ & \\
\hline
\end{tabular}

Data are reported as $n(\%)$ or median (1st-3rd quartile) or otherwise indicated

${ }^{*} p$ values based on Pearson chi-square for categorical variables and the Wilcoxon rank-sum test for continuous variables

Table 2 Mortality rates stratified for risk of mortality and exposure to chlorhexidine oral care

\begin{tabular}{|c|c|c|c|c|}
\hline & High chlorhexidine oral care & Low chlorhexidine oral care & No chlorhexidine oral care & $p$ value \\
\hline All patients $(n=82,274)$ & $571 / 3053(18 \%)$ & $870 / 8080(11 \%)$ & $954 / 71,141(1 \%)$ & $<0.001$ \\
\hline \multicolumn{5}{|l|}{ Risk of mortality category } \\
\hline 1. Minor $(n=53,233)$ & $4 / 450(1 \%)$ & $34 / 2214(2 \%)$ & $53 / 50,569(0.1 \%)$ & $<0.001$ \\
\hline 2. Moderate $(n=19,154)$ & $46 / 790(6 \%)$ & $161 / 3050(5 \%)$ & $254 / 15,314(2 \%)$ & $<0.001$ \\
\hline 3. Major $(n=7417)$ & 133/856 (16\%) & 273/1874 (15\%) & $402 / 4687(9 \%)$ & $<0.001$ \\
\hline 4. Extreme $(n=2470)$ & 388/957 (41\%) & $402 / 942(43 \%)$ & $245 / 571(43 \%)$ & 0.55 \\
\hline
\end{tabular}


Table 3 Adjusted relationships with mortality in the total study cohort $(n=82,274)$

\begin{tabular}{|c|c|c|c|}
\hline & & $\begin{array}{l}\text { Odds ratio } \\
\text { ( } 95 \% \text { confidence interval) }\end{array}$ & $p$ value \\
\hline \multicolumn{4}{|l|}{ Chlorhexidine oral care } \\
\hline No $(0 \mathrm{mg})$ & & Reference & - \\
\hline Low $(\leq 300 \mathrm{mg})$ & & $2.61(2.32-2.92)$ & $<0.001$ \\
\hline High (> 300 mg) & & $2.73(2.35-3.16)$ & $<0.001$ \\
\hline \multicolumn{4}{|l|}{ Risk of mortality category } \\
\hline 1. Minor & & Reference & - \\
\hline 2. Moderate & & $7.35(5.85-9.35)$ & $<0.001$ \\
\hline 3. Major & & $28.77(22.94-36.48)$ & $<0.001$ \\
\hline 4. Extreme & & $180.86(142.63-231.74)$ & $<0.001$ \\
\hline \multicolumn{4}{|l|}{ Major Diagnostic Category (MDC) } \\
\hline MDCO0 & $\begin{array}{l}\text { Pre-MDC (Liver transplant, heart and/or lung transplant, bone mar- } \\
\text { row transplant, tracheotomy, and rest group) }\end{array}$ & Reference & - \\
\hline MDC01 & Diseases and disorders of the nervous system & $0.83(0.63-1.10)$ & 0.19 \\
\hline MDC03 & Diseases and disorders of the ear, nose, mouth, and throat & $0.49(0.32-0.74)$ & $<0.001$ \\
\hline MDC04 & Diseases and disorders of the respiratory system & $1.07(0.81-1.42)$ & 0.62 \\
\hline MDC05 & Diseases and disorders of the circulatory system & $0.44(0.34-0.57)$ & $<0.001$ \\
\hline MDC06 & Diseases and disorders of the digestive system & $0.52(0.39-0.69)$ & $<0.001$ \\
\hline MDC07 & Diseases and disorders of the hepatobiliary system and pancreas & $0.68(0.50-0.91)$ & 0.01 \\
\hline MDC08 & $\begin{array}{l}\text { Diseases and disorders of the musculoskeletal system and connec- } \\
\text { tive tissue }\end{array}$ & $0.53(0.38-0.73)$ & $<0.001$ \\
\hline MDC09 & Diseases and disorders of the skin, subcutaneous tissue, and breast & $0.56(0.38-0.83)$ & 0.004 \\
\hline MDC10 & Endocrine, nutritional, and metabolic diseases and disorders & $0.34(0.22-0.52)$ & $<0.001$ \\
\hline MDC11 & Diseases and disorders of the kidney and urinary tract & $0.30(0.21-0.43)$ & $<0.001$ \\
\hline MDC12 & Diseases and disorders of the male reproductive system & $1.14(0.60-2.05)$ & 0.67 \\
\hline MDC13 & Diseases and disorders of the female reproductive system & $0.63(0.32-1.13)$ & 0.14 \\
\hline MDC16 & $\begin{array}{l}\text { Diseases and disorders of blood, blood-forming organs, immuno- } \\
\text { logical disorders }\end{array}$ & $0.36(0.22-0.58)$ & $<0.001$ \\
\hline MDC17 & $\begin{array}{l}\text { Myeloproliferative diseases and disorders, poorly differentiated } \\
\text { neoplasms }\end{array}$ & $0.52(0.38-0.70)$ & $<0.001$ \\
\hline MDC18 & $\begin{array}{l}\text { Infectious and parasitic diseases and disorders, systemic or unspeci- } \\
\text { fied sites }\end{array}$ & $0.48(0.35-0.65)$ & $<0.001$ \\
\hline MDC19 & Mental diseases and disorders & $0.37(0.17-0.72)$ & 0.01 \\
\hline MDC21 & Injuries, poisonings, and toxic effects of drugs & $0.29(0.18-0.46)$ & $<0.001$ \\
\hline MDC22 & Burns & $0.86(0.38-1.80)$ & 0.71 \\
\hline MDC23 & $\begin{array}{l}\text { Factors influencing health status and other contacts with health } \\
\text { services }\end{array}$ & $0.47(0.29-0.73)$ & 0.001 \\
\hline MDC24 & Multiple significant trauma & $0.31(0.12-0.71)$ & 0.01 \\
\hline MDC25 & Human immunodeficiency virus infection & $1.99(1.23-3.20)$ & 0.005 \\
\hline Age, per year increase & & $1.03(1.02-1.03)$ & $<0.001$ \\
\hline Medical or surgical classification, medical & & $3.19(2.80-3.64)$ & $<0.001$ \\
\hline
\end{tabular}

Results of a logistic regression model without interaction terms. Area under the receiver operating characteristic curve of the test data set (one-third of patients): 0.94

To assess the impact of chlorhexidine oral care on the discriminative power of the logistic regression model we subsequently performed a logistic regression model but without chlorhexidine oral care as covariate. This model had less predictive value $\left(\chi^{2}=307.75\right.$, degrees of free$\mathrm{dom}=2, p<0.001$ ), thereby stressing the importance of chlorhexidine oral care as a predictor of mortality.

\section{Adjusted relationships with mortality according to risk of mortality category}

Table 4 describes the results of the logistic regression model assessing adjusted relationships with mortality according to distinct risk of mortality categories. Among patients with an extreme risk of mortality (category 4) chlorhexidine oral care is not associated with increased 
Table 4 Adjusted relationships with mortality in patients subdivided according to risk of mortality category

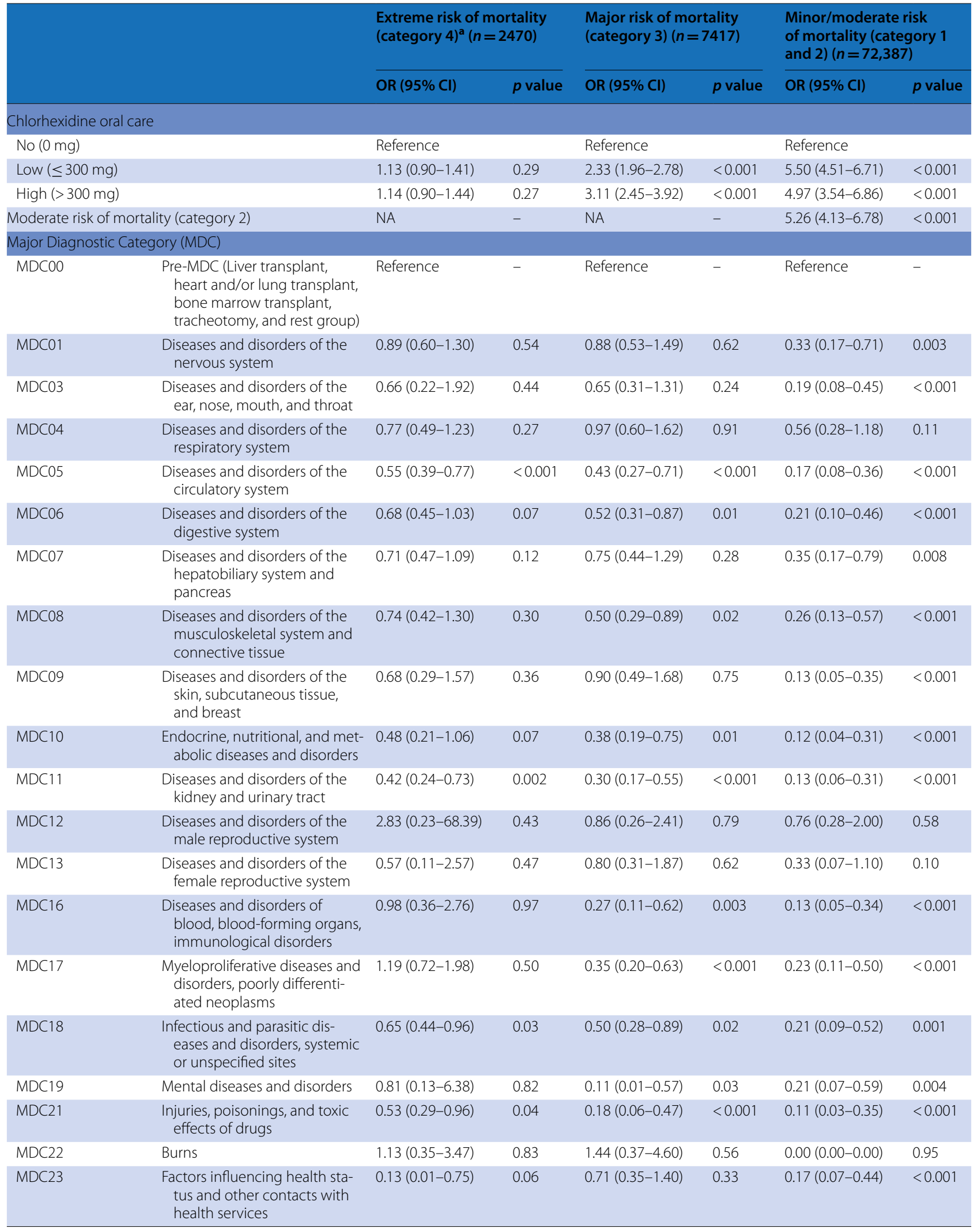


Table 4 continued

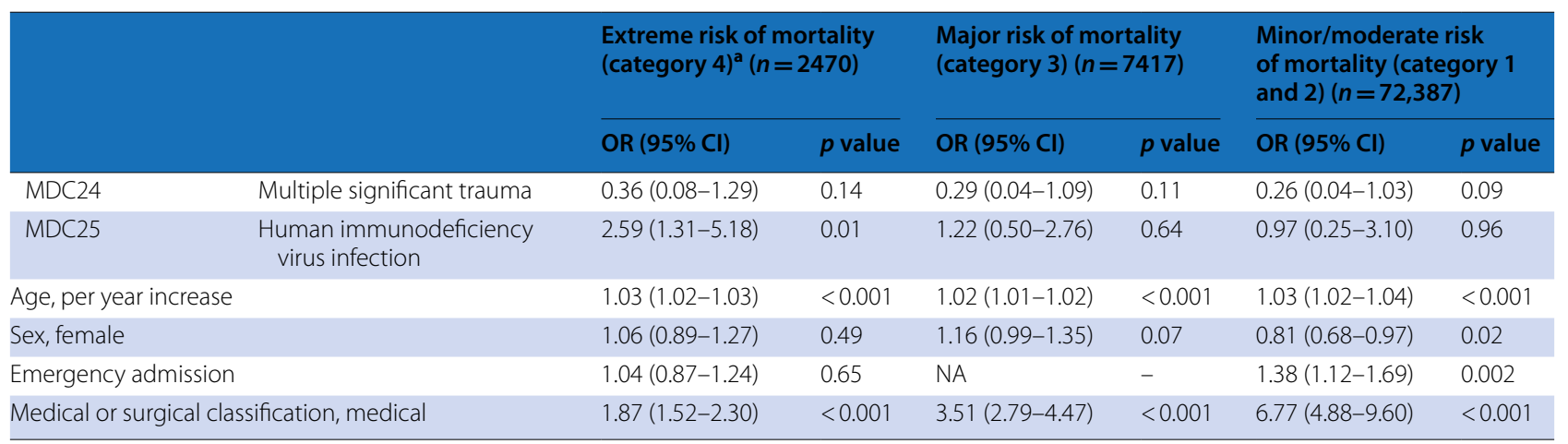

$O R$ odds ratio, $\mathrm{Cl}$ confidence interval, NA not applicable

${ }^{a}$ Full model is shown

mortality. Among patients with a major risk of mortality (category 3 ) chlorhexidine oral care is significantly associated with mortality, as in patients with a minor or moderate risk of mortality (category 1 and 2 ).

\section{Cardiothoracic and vascular surgery patients}

The selection of major cardiothoracic and vascular surgery patients encompassed 1106 patients of which 922 received chlorhexidine oral care $(83.4 \%)$. Mortality in this subgroup was $3.80 \%(n=42)$. Logistic regression analysis revealed that chlorhexidine oral care had no effect on mortality in these patients (chlorhexidine exposure $\leq 300 \mathrm{mg}$ OR 0.70 ; $95 \%$ CI $0.20-2.89 ; p=0.594$, chlorhexidine exposure > $300 \mathrm{mg}$ OR 0.98; 95\% CI 0.29-3.39; $p=0.594)$. Thereafter, we broadened the patient selection to all cardiothoracic and vascular surgery patients. This subgroup included 6334 patients of which 1915 received chlorhexidine (30.2\%) and 156 died (2.47\%). In this patient category the relationship between chlorhexidine oral care and mortality also did not reach statistical significance (chlorhexidine exposure $\leq 300 \mathrm{mg}$ OR 0.96; $95 \%$ CI $0.60-1.55 ; p=0.874$, chlorhexidine exposure $>300 \mathrm{mg}$ OR 1.43; 95\% CI 0.88-2.32; $p=0.146$ ) (Supplementary Appendix 4). Subanalyses for all MDCs are reported in Supplementary Appendix 5.

\section{Mechanical ventilation}

Absolute numbers show that mortality in patients who did and did not receive chlorhexidine oral care is substantially different among the groups with and without mechanical ventilation.

Logistic regression analysis revealed that chlorhexidine oral care had no harmful effect on the group of ventilated patients. After splitting this group according to length of mechanical ventilation a beneficial effect was noted for patients ventilated $\leq 96 \mathrm{~h}$. No effect was observed among patients ventilated for $>96 \mathrm{~h}$.

Chlorhexidine oral care was associated with increased risk of death in patients who did not receive mechanical ventilation and were not admitted to the ICU during their hospitalization. Contrariwise, among non-ventilated ICU patients no deleterious effect of chlorhexidine oral care was observed. Absolute mortality figures and results are provided in Table 5.

\section{Discussion}

This large retrospective, cohort study revealed that chlorhexidine oral care is associated with increased mortality in a general hospitalized population. While the deleterious impact is evident in all risk of mortality categories, the risk of death estimates stepwise increase with lower risk of mortality categories. Among patients undergoing major cardiothoracic and vascular surgery, as well as among patients receiving mechanical ventilation, chlorhexidine oral care was not associated with increased mortality.

This study has limitations. Firstly, this is an observational study and is therefore prone to bias. Also the observational study design did not allow an in-depth analysis of the microbiological effect of chlorhexidine. Secondly, adjustment for diagnostic category might be suboptimal as the categories are broad and may include wide ranges of diseases. However, the assignment of a risk of mortality subclass takes into consideration primary diagnosis, secondary diagnoses, including underlying conditions, age, surgical/medical procedures, as well as interactions between these covariates [21]. This risk of mortality categorization previously proved to correlate strongly with observed mortality in a setting encompassing a broad case-mix [25]. Also, when considering our total cohort, the observed mortality stepwise escalates 
Table 5 Mortality rates according to exposure to chlorhexidine oral care in mechanically ventilated patients

\begin{tabular}{|c|c|c|c|c|c|}
\hline & Survivors, $N=79,879$ & Non-survivors, $N=2395$ & $\%$ non-survivors & OR $(95 \% \mathrm{Cl})$ & $p$ value \\
\hline \multicolumn{6}{|l|}{ Without mechanical ventilation } \\
\hline \multicolumn{6}{|l|}{ No ICU stay } \\
\hline No chlorhexidine oral care $(0 \mathrm{mg})$ & 65,034 & 751 & 1 & Reference & - \\
\hline Low chlorhexidine oral care $(\leq 300 \mathrm{mg})$ & 2375 & 363 & 13 & $4.86(4.17-5.66)$ & $<0.001$ \\
\hline High chlorhexidine oral care (>300 mg) & 584 & 101 & 15 & $3.71(2.84-4.80)$ & $<0.001$ \\
\hline \multicolumn{6}{|l|}{ ICU stay } \\
\hline No chlorhexidine oral care (0 mg) & 4854 & 118 & 2 & Reference & - \\
\hline Low chlorhexidine oral care $(\leq 300 \mathrm{mg})$ & 3244 & 187 & 5 & $0.86(0.63-1.14)$ & 0.27 \\
\hline High chlorhexidine oral care (>300 mg) & 818 & 95 & 10 & $0.95(0.64-1.40)$ & 0.81 \\
\hline \multicolumn{6}{|l|}{ With mechanical ventilation $\leq 96 \mathrm{~h}$} \\
\hline No chlorhexidine oral care (0 mg) & 263 & 65 & 20 & Reference & - \\
\hline Low chlorhexidine oral care ( $\leq 300$ mg) & 1469 & 220 & 13 & $0.58(0.39-0.87)$ & 0.008 \\
\hline High chlorhexidine oral care (> 300 mg) & 687 & 143 & 17 & $0.51(0.34-0.79)$ & 0.003 \\
\hline \multicolumn{6}{|l|}{ With mechanical ventilation $>96 \mathrm{~h}$} \\
\hline No chlorhexidine oral care (0 mg) & 36 & 20 & 36 & Reference & - \\
\hline Low chlorhexidine oral care $(\leq 300 \mathrm{mg})$ & 122 & 100 & 45 & $1.47(0.75-2.91)$ & 0.26 \\
\hline High chlorhexidine oral care (> 300 mg) & 393 & 232 & 37 & $1.11(0.59-2.14)$ & 0.74 \\
\hline
\end{tabular}

$O R$ odds ratio, $\mathrm{Cl}$ confidence interval

with increasing risk of mortality categories. Therefore, we assume that accurate risk adjustment for mortality was achieved. Nevertheless, this study remains a rough epidemiological exercise and risk adjustment might not be accurate for specific subgroups. Thirdly, this study is exclusively based on administrative data and may lack the subtleties of data collected from clinical files. Fourthly, we dichotomized the cumulative chlorhexidine dose. This implies that patients in the group $\leq 300 \mathrm{mg}$ might have been exposed to a single oral care provision (approximately $15 \mathrm{~mL}$ or $18 \mathrm{mg}$ ) as well as to thrice-daily oral care for 6 days. We were not capable of adjusting for the exact length of exposure time. On the same line more specific analyses according to length of stay or length of mechanical ventilation were not possible as the database does not include any time-dependent variables. Finally, all patients were treated with a mouth rinse containing low concentrations of chlorhexidine $(0.05 \%$ or $0.12 \%)$. We were unable to compare to outcomes associated with higher concentrations such as $0.2 \%, 1 \%$, and $2 \%[27,28]$. Also, the number of patients receiving oral care with the $0.05 \%$ chlorhexidine solution was too low to allow a meaningful comparison with those exposed to the $0.12 \%$ concentration.

The adverse effect of chlorhexidine oral care on outcome is greater among patients with an inherently more favorable prognosis. This might be explained by a relatively greater attributable mortality due to chlorhexidine in moderately as compared to severely ill patients in which a substantial proportion of mortality can be attributed to disease severity.

In the subgroup of patients ventilated $<96 \mathrm{~h}$ a small beneficial effect was observed among those exposed to chlorhexidine, an observation that contradicts previous reports [16-18]. This result, however, must be interpreted with caution. A vast majority of ventilated patients did receive chlorhexidine oral care. Patients not receiving chlorhexidine oral care include those who died soon after ICU admission. As such, survival bias might have played a role in favor of those exposed to chlorhexidine.

Chlorhexidine oral care significantly reduces the risk of nosocomial pneumonia in cardiac surgery patients. In the meta-analysis by Klompas et al. the trend towards increased risk of death was not observed in cardiac surgery studies [16]. Our data confirm this finding albeit that we enlarged this group to patients undergoing major cardiothoracic and vascular surgery.

Whether the absence of an increased risk of mortality can be attributed to a reduced risk of pneumonia could not be demonstrated. Therefore, it remains hard to explain why cardiac surgery patients are spared from an increased risk of mortality when exposed to chlorhexidine oral care.

The absence of a clear pathogenic mechanism leading to increased risk of death renders these data hard to interpret. In intubated patients micro-aspiration of subglottic secretions containing chlorhexidine has been previously proposed as a potential mechanism [16]. Indeed, a 
fatal case of acute respiratory distress syndrome has been reported in an elderly patient who accidentally ingested $200 \mathrm{~mL}$ of $5 \%$ chlorhexidine after which the patient aspirated gastric contents [29]. Additionally, experimental animal data have demonstrated the acute pulmonary toxic effects of chlorhexidine [30]. In both circumstances, however, the exposure to chlorhexidine was much higher than what might be expected in terms of silent microaspiration along the folds in an inflated endotracheal tube cuff [6]. Moreover, our observation undermines the aforementioned hypothesis as the association between chlorhexidine and increased mortality was overt in nonventilated patients, while absent in ventilated patients. Life-threatening anaphylactic reactions following exposure of chlorhexidine at mucous membranes have been described with the use of chlorhexidine-impregnated intravascular catheters and gel for bladder catheterization [31-33]. Yet, such reactions are so explicit that they can hardly serve as a possible explanation for an adverse event that remained undetected for decades. Exposing the oral mucosa to chlorhexidine reduces colonization with important respiratory pathogens but undoubtedly affects the normal oral microbiome as well [34]. However, while the role of the oral microbiome to prevent local and systemic disease is proven, it seems unlikely that disturbing the oral microbiota might give occasion to potentially lethal complications in the short term [35].

Although only marginally supported by evidence, the practice of chlorhexidine oral care has been generalized to a variety of patient populations beyond cardiac surgery. Considering the present data, and those provided by Klompas et al., we believe that chlorhexidine oral care in non-cardiac surgery patients should be re-evaluated. One might indeed question whether the increased risk of mortality, albeit without a clear pathogenic mechanism, outweighs the marginal effect in pneumonia risk reduction. Likewise, since we observed the greatest associations among patients with a more favorable risk profile, one might question the safety of chlorhexidine mouth washes in ambulatory settings (e.g., dentistry) where this has been practiced for decades without any indication towards serious toxicity, but also without any monitoring of serious adverse events. In any case, these new data further fuel the controversy on a practice that was thought to be safe for many years. The reason why this increased mortality risk could remain undetected is undoubtedly the low occurrence rate of death as well as the powering of clinical trials to detect differences in pneumonia, rather than in mortality. Future trials with chlorhexidine oral care, in any concentration studied, should be powered to detect differences in mortality. Furthermore, in order to bring insight into the pathogenic mechanism, careful observation and stricter recording of adverse events seem required.
In conclusion, in a general hospital population chlorhexidine oral care is significantly associated with increased mortality. The risk estimates for death are higher among patients with an intrinsically more favorable prognosis. In cardiac surgery patients and patients on mechanical ventilation no effect on mortality was found. These findings incite reconsideration of the practice of chlorhexidine oral care in patient populations for which an evidence-based benefit is lacking.

\section{Electronic supplementary material}

The online version of this article (https://doi.org/10.1007/s00134-018-5171-3) contains supplementary material, which is available to authorized users.

\section{Author details \\ ${ }^{1}$ Strategic Policy Cell, Ghent University Hospital, Corneel Heymanslaan 10, 9000 Ghent, Belgium. ${ }^{2}$ Department of Data Analysis and Mathematical Mode- ling, Ghent University, Coupure Links 653, 9000 Ghent, Belgium. ${ }^{3}$ Department of Public Health, Ghent University, Corneel Heymanslaan 10, 9000 Ghent, Belgium. ${ }^{4}$ General Internal Medicine, Ghent University Hospital, Corneel Hey- manslaan 10, 9000 Ghent, Belgium. ${ }^{5}$ Department of Internal Medicine, Ghent University, Campus UZ Gent, Corneel Heymanslaan 10, 9000 Ghent, Belgium. ${ }^{6}$ Burns, Trauma and Critical Care Research Centre, Centre for Clinical Research, Faculty of Medicine, The University of Queensland, Brisbane, Australia.}

\section{Author contributions}

Contributed to conception and design: Deschepper, Blot. Contributed to acquisition of data: Deschepper, Vogelaers, Eeckloo, Blot. Contributed to analyses of data: Deschepper, Waegeman, Blot. Contributed to interpretation of data: Deschepper, Waegeman, Vogelaers, Blot. Drafting the work: Deschepper, Blot. Revising the paper for important intellectual content: Vogelaers, Waegeman, Eeckloo. Final approval of the version submitted: all authors. Agreement to be accountable for all aspects of the work in ensuring that questions related to the accuracy or integrity of any part of the work are appropriately investigated and resolved: all authors.

\section{Funding}

None. SB holds a research mandate of the Special Research Fund at Ghent University.

\section{Compliance with ethical standards}

\section{Conflicts of interest}

All authors declare that there are no potential conflicts of interest to disclose.

\section{Compliance with Ethics Guidelines}

The study was approved by the ethics committee at Ghent University Hospital (Belgian registration no. B670201731386).

\section{Open Access}

This article is distributed under the terms of the Creative Commons Attribution-NonCommercial 4.0 International License (http://creativecommons.org/ licenses/by-nc/4.0/), which permits any noncommercial use, distribution, and reproduction in any medium, provided you give appropriate credit to the original author(s) and the source, provide a link to the Creative Commons license, and indicate if changes were made.

Received: 9 January 2018 Accepted: 7 April 2018

Published online: 9 May 2018

\section{References}

1. Melsen WG, Rovers MM, Bonten MJM (2009) Ventilator-associated pneumonia and mortality: a systematic review of observational studies. Crit Care Med 37:2709-2718 
2. Muscedere JG, Martin CM, Heyland DK (2008) The impact of ventilatorassociated pneumonia on the Canadian health care system. J Crit Care 23:5-10. https://doi.org/10.1016/j.jcrc.2007.11.012

3. Blot $\mathrm{S}$, Koulenti D, Dimopoulos $\mathrm{G}$ et al (2014) Prevalence, risk factors, and mortality for ventilator-associated pneumonia in middle-aged, old, and very old critically ill patients. Crit Care Med 42:601-609. https://doi. org/10.1097/01.ccm.0000435665.07446.50

4. Lorente L, Blot S, Rello J (2007) Evidence on measures for the prevention of ventilator-associated pneumonia. Eur Respir J 30:1193-1207. https:// doi.org/10.1183/09031936.00048507

5. Lorente L, Blot S, Rello J (2010) New issues and controversies in the prevention of ventilator-associated pneumonia. Am J Respir Crit Care Med 182:870-876. https://doi.org/10.1164/rccm.201001-0081Cl

6. Blot SI, Poelaert J, Kollef M (2014) How to avoid microaspiration? A key element for the prevention of ventilator-associated pneumonia in intubated ICU patients. BMC Infect Dis 14:119. https://doi. org/10.1186/1471-2334-14-119

7. Nseir S, Lorente L, Ferrer M et al (2015) Continuous control of tracheal cuff pressure for VAP prevention: a collaborative meta-analysis of individual participant data. Ann Intensive Care 5:43. https://doi.org/10.1186/ s13613-015-0087-3

8. Maertens B, Blot K, Blot S (2018) Prevention of ventilator-associated and early postoperative pneumonia through tapered endotracheal tube cuffs: a systematic review and meta-analysis of randomized controlled trials. Crit Care Med 46:316-323. https://doi.org/10.1097/ CCM.0000000000002889

9. Labeau SO, Van de Vyver K, Brusselaers N et al (2011) Prevention of ventilator-associated pneumonia with oral antiseptics: a systematic review and meta-analysis. Lancet Infect Dis 11:845-854. https://doi.org/10.1016/ S1473-3099(11)70127-X

10. American Thoracic Society, Infectious Diseases Society of America (2005) Guidelines for the management of adults with hospital-acquired, ventilator-associated, and healthcare-associated pneumonia. Am J Respir Crit Care Med 171:388-416. https://doi.org/10.1164/rccm.200405-644ST

11. Healthcare Infection Control Practices Advisory Committee, Centers for Disease Control and Prevention (US) (2004) Guidelines for preventing health-care-associated pneumonia, 2003 recommendations of the CDC and the Healthcare Infection Control Practices Advisory Committee. Respir Care 49:926-939

12. Institute for Healthcare Improvement (2012) How-to guide: prevent ventilator-associated pneumonia. Institute for Healthcare Improvement, Cambridge

13. Mohr NM, Pelaez Gil CA, Harland KK et al (2015) Prehospital oral chlorhexidine does not reduce the rate of ventilator-associated pneumonia among critically ill trauma patients: a prospective concurrent-control study. J Crit Care 30:787-792. https://doi.org/10.1016/j.jcrc.2015.03.017

14. Hollaar V, van der Maarel-Wierink C, van der Putten G-J et al (2015) Effect of daily application of a $0.05 \%$ chlorhexidine solution on the incidence of (aspiration) pneumonia in care home residents: design of a multicentre cluster randomised controlled clinical trial. BMJ Open 5:e007889. https:// doi.org/10.1136/bmjopen-2015-007889

15. Sharif-Abdullah SSB, Chong MC, Surindar-Kaur SS et al (2016) The effect of chlorhexidine in reducing oral colonisation in geriatric patients: a randomised controlled trial. Singap Med J 57:262-266. https://doi. org/10.11622/smedj.2016091

16. Klompas M, Speck K, Howell MD et al (2014) Reappraisal of routine oral care with chlorhexidine gluconate for patients receiving mechanical ventilation: systematic review and meta-analysis. JAMA Intern Med 174:751-761. https://doi.org/10.1001/jamainternmed.2014.359

17. Price R, MacLennan G, Glen J, SuDDICU Collaboration (2014) Selective digestive or oropharyngeal decontamination and topical oropharyngeal chlorhexidine for prevention of death in general intensive care: systematic review and network meta-analysis. BMJ 348:g2197-g2197. https:// doi.org/10.1136/bmj.g2197
18. Klompas M, Li L, Kleinman K et al (2016) Associations between ventilator bundle components and outcomes. JAMA Intern Med 176:1277-1283. https://doi.org/10.1001/jamainternmed.2016.2427

19. Torres A, Niederman MS, Chastre J et al (2017) International ERS/ESICM/ ESCMID/ALAT guidelines for the management of hospital-acquired pneumonia and ventilator-associated pneumonia: guidelines for the management of hospital-acquired pneumonia (HAP)/ventilatorassociated pneumonia (VAP) of the European Respiratory Society (ERS), European Society of Intensive Care Medicine (ESICM), European Society of Clinical Microbiology and Infectious Diseases (ESCMID) and Asociación Latinoamericana del Tórax (ALAT). Eur Respir J 50:1700582. https://doi. org/10.1183/13993003.00582-2017

20. Centers for Disease Control and Prevention (2011) National Center for Health Statistics. International Classification of Diseases, Ninth Revision, Clinical Modification (ICD-9-CM). https://www.cdc.gov/nchs/icd/icd9cm. htm. Accessed 24 May 2017

21. 3M Health Information Systems (2003) All Patient Refined Diagnosis Related Groups (APR-DRGs), Version 20.0, methodology overview. https:// www.hcup-us.ahrq.gov/db/nation/nis/APR-DRGsV20MethodologyOverviewandBibliography.pdf. Accessed 23 May 2017

22. Iezzoni LI, Ash AS, Shwartz M et al (1995) Predicting who dies depends on how severity is measured: implications for evaluating patient outcomes. Ann Intern Med 123:763-770

23. Messina G, Forni S, Collini F et al (2015) Short-term adjusted outcomes for heart failure. Heart Int 10:e1-e5. https://doi.org/10.5301/heartint.5000220

24. De Marco MF, Lorenzoni L, Addari P, Nante N (2002) Evaluation of the capacity of the APR-DRG classification system to predict hospital mortality. Epidemiol Prev 26:183-190

25. Baram D, Daroowalla F, Garcia R et al (2008) Use of the All Patient RefinedDiagnosis Related Group (APR-DRG) risk of mortality score as a severity adjustor in the medical ICU. Clin Med Circ Respir Pulm Med 2:19-25

26. Bender R, Blettner M (2002) Calculating the "number needed to be exposed" with adjustment for confounding variables in epidemiological studies. J Clin Epidemiol 55:525-530

27. Plantinga NL, Wittekamp BHJ, Leleu K et al (2016) Oral mucosal adverse events with chlorhexidine 2\% mouthwash in ICU. Intensive Care Med 42:620-621. https://doi.org/10.1007/s00134-016-4217-7

28. Zand F, Zahed L, Mansouri P et al (2017) The effects of oral rinse with $0.2 \%$ and $2 \%$ chlorhexidine on oropharyngeal colonization and ventilator associated pneumonia in adults' intensive care units. J Crit Care. https:// doi.org/10.1016/j.jcrc.2017.02.029

29. Hirata K, Kurokawa A (2002) Chlorhexidine gluconate ingestion resulting in fatal respiratory distress syndrome. Vet Hum Toxicol 44:89-91

30. Xue Y, Zhang S, Yang Y et al (2011) Acute pulmonary toxic effects of chlorhexidine (CHX) following an intratracheal instillation in rats. Hum Exp Toxicol 30:1795-1803. https://doi.org/10.1177/0960327111400104

31. Stephens R, Mythen M, Kallis P et al (2001) Two episodes of life-threatening anaphylaxis in the same patient to a chlorhexidine-sulphadiazinecoated central venous catheter. $\mathrm{Br} J$ Anaesth 87:306-308

32. Jee R, Nel L, Gnanakumaran G et al (2009) Four cases of anaphylaxis to chlorhexidine impregnated central venous catheters: a case cluster or the tip of the iceberg? Br J Anaesth 103:614-615. https://doi.org/10.1093/bja/ aep248

33. Parkes AW, Harper N, Herwadkar A, Pumphrey R (2009) Anaphylaxis to the chlorhexidine component of Instillagel: a case series. Br J Anaesth 102:65-68. https://doi.org/10.1093/bja/aen324

34. Tuon FF, Gavrilko O, de Almeida S et al (2017) Prospective, randomised, controlled study evaluating early modification of oral microbiota following admission to the intensive care unit and oral hygiene with chlorhexidine. J Glob Antimicrob Resist 8:159-163. https://doi.org/10.1016/j. jgar.2016.12.007

35. Sampaio-Maia B, Caldas IM, Pereira ML et al (2016) The oral microbiome in health and its implication in oral and systemic diseases. Adv Appl Microbiol 97:171-210. https://doi.org/10.1016/bs.aambs.2016.08.002 